\title{
Direct-Write Dispenser Printing for Rapid Antenna Prototyping on Thin Flexible Substrates
}

\author{
Mahmoud Wagih, Graduate Student Member, IEEE \\ School of Electronics and Computer Science, University of Southampton, mahm1g15@ecs.soton.ac.uk
}

\begin{abstract}
Rapid prototyping of antennas is crucial to validation of simulation models when designing conformal antennas on unusual substrates such as polymers and textiles. This paper presents direct-write dispenser printing, using a commercial Printed Circuit Board (PCB) printer, as a simple mean of prototyping planar antennas on ultra-thin $(25 \mu \mathrm{m})$ flexible Polyimide substrates. Two Coplanar Waveguide (CPW) monopole antennas have been designed for the $2.4 \mathrm{GHz}$ band and fabricated using dispenser printing and standard photolithography. The impedance bandwidth and gain of both antennas has been compared and the printed prototype was found to match the performance of the etched antenna within a $2.6 \%$ and $2.3 \%$ margin respectively, as well as matching the full-wave 3D simulation of the connectorized antennas. Based on the measured performance of the printed antenna, the potential of utilising commercial dispenser printers to prototype and manufacture low-volume antennas for low-cost unobtrusive Internet of Things applications is demonstrated.
\end{abstract}

Index Terms-Antenna, Coplanar Waveguide, Direct-Write, Printed Electronics, Wearable Electronics

\section{INTRODUCTION}

Additive manufacturing is increasingly attracting interest in Radio Frequency (RF) and microwave applications. Passive components including antennas, filters, and transmission lines have been previously presented using printed conductive inks on a variety of substrates such as textiles, 3D printed enclosures, and stretchable materials [1], [2]. Moreover, complete systems such as RF energy harvesters have been realised using a combination of 3D and inkjet printing [3], for next generation low-cost pervasive connected Internet of Things (IoT) networks.

The main limitations of printed RF components are the performance, compared to their etched counterparts, and the throughput in mass manufacturing. The throughput of printing remains a challenge in commercial-scale manufacturing; limiting inkjet and direct-write printing to rapid research and development prototypes [4]. Furthermore, inkjet and screen printing of conductive traces require fine tuning of multiple parameters such as the ink's viscosity and the jetting mechanism, which may not be feasible within the development cycle of an antenna, and requires substantial printed electronics experience [5]. To add, the reliability of printed traces and the incompatibility of most conductive inks with standard soldering temperatures are significant drawbacks of printed

This work was supported by the European Commission through the project EnABLES: European Infrastructure Powering the Internet of Things, funded under H2020-EU.1.4.1.2.
RF electronics compared to etched flexible copper laminates [6].

Flexible copper laminates can be laser-cut, laser-milled or etched using standard photolithography techniques to create high efficiency flexible microwave and mmWave components [7], achieving up to $25 \%$ higher radiation efficiency using laser-etched Liquid Crystal Polymer (LCP) laminate compared to inkjet printing on the same substrate. Moreover, etched and laser-ablated flexible copper sheets have been used to fabricate textile antennas of up to $77 \%$ and $41 \%$ radiation efficiency at $28 \mathrm{GHz}$ [8] and $60 \mathrm{GHz}$ respectively [9]. In addition to improved conductivity, the added thermal and mechanical reliability of etched circuits enable mounting fine-pitch components using standard Printed Circuit Board (PCB) assembly tools to realise a flexible wireless transmitter at $2.4 \mathrm{GHz}$ [10]. Nevertheless, the main limitation of photolithography is the high initial cost and setup time associated with obtaining an ultraviolet exposure mask, making it an inefficient process for rapid prototyping and validating numerical models.

Direct-write dispenser printing has been utilised to create traces of good resolution down to millimetre-sized features while not requiring detailed optimisation of the process [11]. While direct-written antennas have been reported on textiles [4], [12] and conformal substrates [13], direct-writing of antennas did not become apparent as a standard antenna fabrication technique and has only been utilized for sensors and DC or low-frequency circuits such as LEDs [11] and energy harvesters [14]. Moreover, detailed studies of dispenser printed antennas have been limited to Radio Frequency Identification (RFID) and sub-1 GHz applications [4].

This work presents dispenser printing as a rapid antenna prototyping method, which does not require prior knowledge of circuit printing optimisation techniques, as a step in a planar flexible antenna design flow and for validation of simulation models. A commercially-available low-cost dispenser printer is utilised to print a $2.4 \mathrm{GHz}$ Coplanar-Waveguide (CPW) monopole antenna as a prototype for a photolithographyetched antenna. The performance of the printed antenna is compared to that of the numerical model and the etched antenna in order to evaluate the validity of printed antenna prototypes. The paper is the first detailed experimental evaluation of a dispenser printed antenna independent of geometry and substrate, validating the approach of direct-write printing as a prototyping method and comparing its performance to standard photolithography. 
This paper is structured as follows, section II describes the fabrication processes of the antenna, section III presents the CPW antenna's design and simulation and section IV compares the performance of the prototypes and evaluates the validity of the approach.

\section{DiRect-Write Dispenser Printing}

Dispenser printing is a thick-film deposition technique widely used in fabricating devices such as sensors and energy harvesters [14]. It is distinguished from screen printing by not requiring a screen to produce the design, which enables "direct-writing" of the Computer-Aided Design (CAD) drawing onto the substrate. Conductive material-waste, often the biggest cost in additively manufactured circuits, is minimized compared to screen printing and etching as only the trackpatterns are deposited on the substrate.

The printer utilized in this study is the Voltera V-one PCBprototyping printer, which enables "plug-and-play" replacement of conductive inks or solder paste cartridges, along with minimal optimization or calibration of the ink's deposition settings; eliminating the need for expertise in printing optimisation by the circuit designer. The minimum supported track width is $0.3 \mathrm{~mm}$ with a clearance of $0.65 \mathrm{~mm}$ between tracks. The conductive ink, composed of $>70 \%$ silver particles, has a bulk resistivity of $9.5 \times 10^{-7} \Omega \mathrm{m}$, compared to $1.7 \times 10^{-8}$ of standard copper sheets.

The antenna CAD layout is loaded to the printer in Gerber format, the printing plan is then automatically generated. Prior to printing, the thickness of the substrate is measured automatically using a measurement probe. Hence, a certain level of tolerance to changes in thickness is guaranteed, which makes this process compatible with flexible substrates. The silver ink particles are then jetted onto the substrate to form the track's patterns. While it is possible to then cure the ink on the printer's own heating pad, due to the non-planarity of the flexible substrate, the uncured ink may be smeared when placed facing the printer's own heating pad. Therefore, the printed antenna has been cured using a standard soldering hot plate, set to $170^{\circ} \mathrm{C}$, for 40 minutes. The sample is then allowed to cool-down at room temperature before the SMA connector is soldered.

As the ink can only withstand temperatures up to $230 \mathrm{deg}$ (a standard reflow profile peak temperature), a special lowtemperature solder alloy has been used to solder the SMA. Fig. 1 shows the fabrication steps of the dispenser-printed antenna, and a photograph of the prototype-under-test. The total fabrication time of the antenna is less than 1 hour from finalising the CAD layout to testing using a Vector Network Analyser (VNA).

\section{Antenna Design And Simulation}

The proposed antenna design in this study, to evaluate the validity of dispenser-printing as an antenna prototyping tool, is a simple CPW monopole. The antenna has been designed and simulated in CST Microwave Studio, to achieve resonance at $2.4 \mathrm{GHz}$, the most popular Industrial Scientific Medical (a)

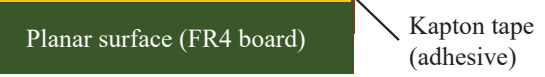

(b)
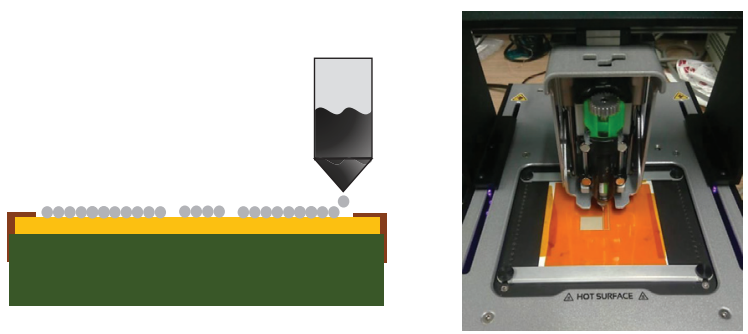

(c)

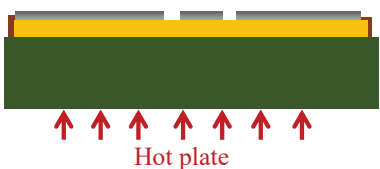

(d)

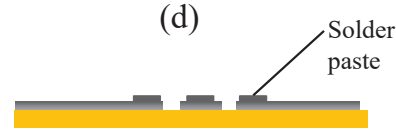

Hot plate

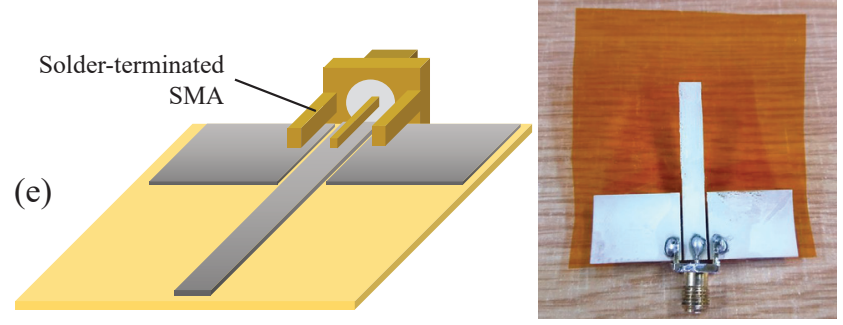

Fig. 1. Diagram of the direct-write antenna fabrication steps: (a) the Polyimide substrate adhered to a planar surface using Kapton tap, (b) diagram and photograph deposition of the silver-ink particles using the computer-controlled nozzle, (c) thermal curing of the ink, (d) deposition of solder paste, (e) mounting of the RF connector on the printed CPW trace and photograph of the fabricated antenna.

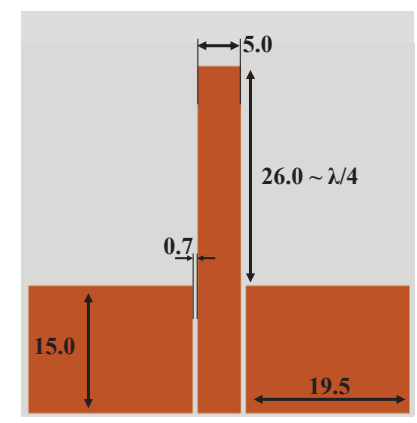

Fig. 2. Antenna layout and dimensions in $\mathrm{mm}$.

(ISM)-band for body area networks and IoT applications. Fig. 2 shows the layout and dimensions of the proposed antenna.

CST's lossy copper model $\left(5.8 \times 10^{-} 8 \Omega \mathrm{m}\right)$ has been used for the conductor traces, Kapton polyimide has been modelled as a dielectric material of $\epsilon_{r}=3.2$ and $\tan \delta=0.02$. Fig. 3 shows the simulated reflection coefficient of the CPW monopole, with and without the SMA connector. Based on CST's farfield monitors around $2.4 \mathrm{GHz}$, it is expected that the antenna maintains a stable gain of $2.1 \mathrm{~dB}$ across the full band, the gain plot is shown on the secondary axis of Fig. 3. 


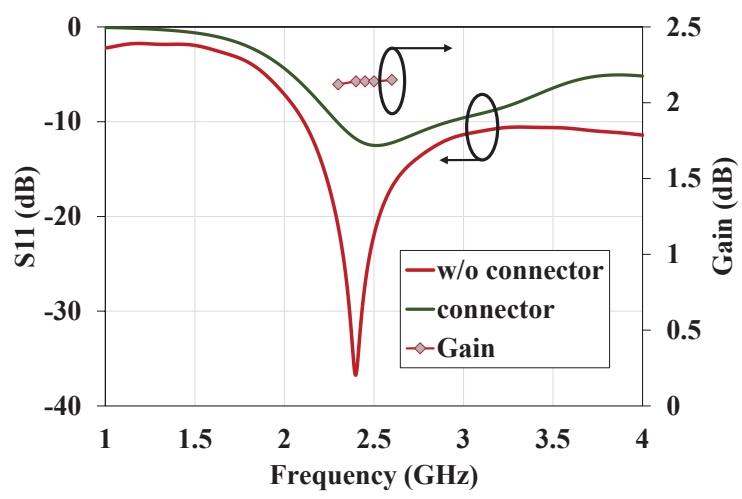

Fig. 3. Simulated reflection coefficient and gain of the antenna with and without including the SMA connector in the 3D model

\section{ETCHED AND PRINTED ANTENNA COMPARISON}

\section{A. Printed Antenna Evaluation}

The first step in identifying the limitations of printing as a mean of prototyping antennas is analysing the geometry of the fabricated structure and observing the discrepancies between the design and the fabricated prototype. While features such as surface roughness may only manifest in insertion losses and subsequently antenna radiation efficiency, variations in critical dimensions, such as the gap size of a CPW, can vary the input impedance significantly. A Nikon $\times 5$ optical microscope has been used to visually check the printed antenna for manufacturing defects; Fig. 4 shows micrographs of the antenna

Observing the non-uniformities seen in Fig. 4-a, d and f, they can all be attributed to the ink dispensing mechanism, where certain ink droplets or lines were not deposited, resulting in non-uniform thickness and in some cases slots in large conductive planes. This is due to the dispenser head requiring additional pressure on the piston, which is normally applied automatically once the ink pressure drops bellow a certain threshold.

\section{B. Etched and Printed Antenna Measurements}

As this work proposes direct-write printing as a method of prototyping antennas prior to mass-production using photolithography, the antenna design has been etched on a $25 \mu \mathrm{m}$ polyimide copper laminate; using the process described in [8], in order to compare its performance to the printed antenna. The printed and etched antennas were connected using the same SMA connectors and measured using a ZVB4 VNA calibrated using a Through, Open, Short and Match (TOSM), from 1 to $4 \mathrm{GHz}$. Fig. 5 shows the measured reflection coefficient of the two antennas.

In order to measure the antenna's gain, two identical antennas were connected to the VNA's two ports, at $1 \mathrm{~m}$ separation from each other. The forward transmission $\left(\mathrm{S}_{21}\right)$ was used to calculate the gain of the antennas after calculating the path losses using the Friis free space propagation model. While this technique may result in inaccurate gain measurements,

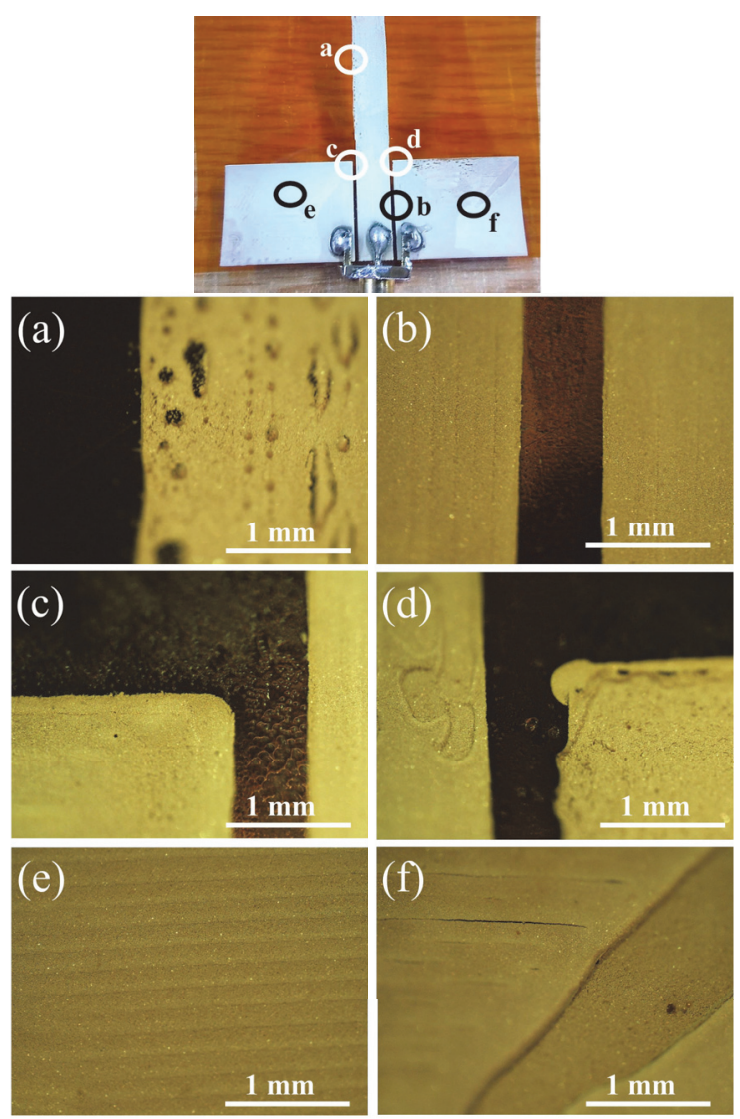

Fig. 4. Micrographs of the printed CPW antenna and their respective location on the antenna, (a): gaps and non-uniform droplets due to variation in the ink dispensing density, (b): uniform resolution of the CPW gap, (c): curvature of a square ground plane edge, (d): smeared ink and non-uniform deposition of a square edge, (e): correctly deposited and cured filling pattern, (f): a conductor trench caused by deposition of lower ink density.

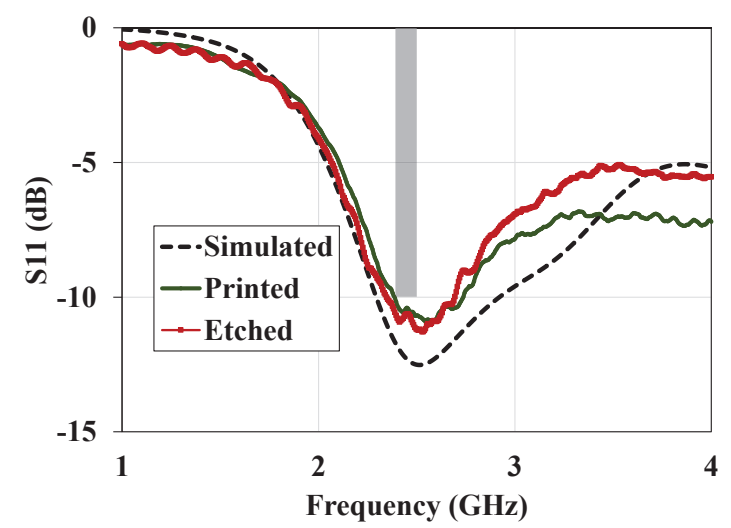

Fig. 5. Measured reflection coefficient $\left(\mathrm{S}_{11}\right)$ of the etched and printed antenna, and the simulated $\mathrm{S}_{11}$, showing a close agreement in around the $2.4 \mathrm{GHz}$ band.

due to additional reflection from nearby objects and multipath effects, the relative gain of both antennas will not be affected due to both being tested under the same conditions. As the purpose of this study is to evaluate the similarity of the two fabrication techniques (printing and etching) and not 
TABLE I

MEASURED ANTENNA CHARACTERISTICS OF THE PRINTED AND ETCHED CONNECTORIZED ANTENNAS

\begin{tabular}{cccc}
\hline & CST Model & Printed Antenna & Etched Antenna \\
\hline Minimum $\mathrm{S}_{11}$ & -12.51 & -10.9 & -11.2 \\
\hline $\mathrm{S}_{11}$ BW $(\mathrm{GHz})$ & $2.31-2.91$ & $2.39-2.73$ & $2.34-2.7$ \\
\hline Realized Gain $(\mathrm{dB})$ & 1.9 & 1.73 & 1.77 \\
\hline
\end{tabular}

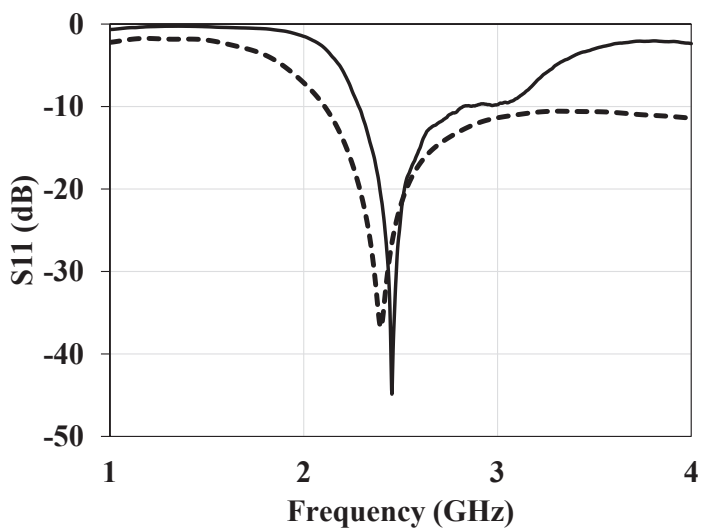

Fig. 6. De-embedded measured reflection coefficient (solid) agreement with the simulated (dashed) at $2.4 \mathrm{GHz}$.

evaluating the antenna design or the performance of a certain antenna, tolerances in the exact gain values are acceptable as the relative gain is the main parameter of interest. Table I gives a summary of the measured antenna parameters and compares them to those of the CST model.

The main discrepancy between the etched and the printed antenna's measured $S_{11}$ is the lower reflection at higher frequencies (3.5 to $4 \mathrm{GHz}$ ), this can be attributed to the miniature imperfections in the printing pattern, such as Fig. 4a and 4-f, resulting in slot-like behaviour due to non-uniform ink-deposition. Moreover, the more broadband behaviour of the printed antenna can be attributed to the more rounded edge and the non-uniform ground plane edge (Fig. 4-d) acting like fractal elements increasing the bandwidth of the antenna. However, both antennas agree from 2 to $2.8 \mathrm{GHz}$ which validates the printed prototype around the frequency band of interest. Furthermore, the measured gain of both antennas at $2.4 \mathrm{GHz}$ closely agrees, the $0.13 \mathrm{~dB}$ difference compared to the simulated model could be attributed to additional losses in the substrate and the connectors.

As a final step in verifying the performance of the printed antenna, and validating the simulation model, the measured results the 3D model of an SMA connector to CPW transition has been simulated in CST and used to de-embed the measured s-parameters of the antenna. Fig. 6 shows the de-embedded $S_{11}$ of the printed, closely matching with the simulated results in magnitude, around $2.4 \mathrm{GHz}$.

\section{CONCLUSION}

This paper has presented detailed evaluation and analysis of direct-write dispenser printing, using a low-cost commerciallyavailable $\mathrm{PCB}$ printer, as a method of prototyping antennas for flexible substrates. It is demonstrated that the performance of the printed antenna, on an ultra-thin substrate, matches the etched copper antenna both in terms of measured gain, reflection coefficient and agreement with the simulated results, implying the suitability of dispenser printing as a substitute for low-volume photolithography prototyping.

Future work includes investigating the insertion losses in a dispenser printed transmission line, and exploring the potential of dispenser printing as a mean of prototyping and realising mmWave antennas of small feature size along with evaluation of in-house direct-write printers.

\section{REFERENCES}

[1] J. Kimionis, M. Isakov, B. S. Koh, A. Georgiadis, and M. M. Tentzeris, "3d-printed origami packaging with inkjet-printed antennas for rf harvesting sensors," IEEE Transactions on Microwave Theory and Techniques, vol. 63, no. 23, pp. 4521 - 4532, 2015.

[2] S. An, A. Meredov, and A. Shamim, "Flexible, stretchable and washable filter printed directly on textile," in 2018 48th European Microwave Conference (EuMC), 2018

[3] T.-H. Lin, S. N. Daskalakis, A. Georgiadis, and M. M. Tentzeris, "Achieving fully autonomous system-on-package designs: An embedded-on-package $5 \mathrm{~g}$ energy harvester within $3 \mathrm{~d}$ printed multilayer flexible packaging structures," in 2019 IEEE MTT-S International Microwave Symposium (IMS), 2019.

[4] J. M. Hoey, M. T. Reich, A. Halvorsen, D. Vaselaar, K. Braaten, M. Maassel, I. S. Akhatov, O. Ghandour, P. Drzaic, and D. L. Schulz, "Rapid prototyping rfid antennas using direct-write," IEEE Transactions on Advanced Packaging, vol. 32, no. 4, pp. 809 - 815, 2009.

[5] W. G. Whittow, A. Chauraya, J. C. Vardaxoglou, Y. Li, R. Torah, K. Yang, S. Beeby, and J. Tudor, "Inkjet-printed microstrip patch antennas realized on textile for wearable applications," IEEE Antennas and Wireless Propagation Letters, vol. 13, pp. 71-74, 2014.

[6] A. Komolafe, R. Torah, Y. Wei, H. NunesMatos, M. Li, D. Hardy, T. Dias, M. Tudor, and S. Beeby, "Integrating flexible filament circuits for etextile applications," Advanced Materials Technologies, vol. 4, no. 7, 2019.

[7] S. F. Jilani, M. O. Munoz, Q. H. Abbasi, and A. Alomainy, "Millimeterwave liquid crystal polymer based conformal antenna array for $5 \mathrm{~g}$ applications," IEEE Antennas and Wireless Propagation Letters, vol. 18, no. 1, 2019.

[8] M. Wagih, A. S. Weddell, and S. Beeby, "Millimeter-wave textile antenna for on-body rf energy harvesting in future $5 \mathrm{~g}$ networks," in 2019 IEEE Wireless Power Transfer Conference (WPTC), 2019.

[9] N. Chahat, M. Zhadobov, S. A. Muhammad, L. L. Coq, and R. Sauleau, "60-ghz textile antenna array for body-centric communications," IEEE Transactions on Antennas and Propagation, vol. 61, 4, pp. 1816 - 1824, 2013.

[10] M. Wagih, Y. Wei, and S. Beeby, "Flexibe $2.4 \mathrm{ghz}$ sensor node for body area networks with a compact high-gain planar antenna," IEEE Antennas and Wireless Propagation Letters, vol. 17, 12, 2018.

[11] Y. Zheng, Z. He, Y. Gao, and J. Liu, "Direct desktop printed-circuitson-paper flexible electronics," Scientific Reports, vol. 3, 2013.

[12] Y. Li, R. Torah, S. Beeby, and J. Tudor, "Fully direct-write dispenser printed dipole antenna on woven polyester cotton fabric for wearable electronics applications," Electronics Letters, vol. 51, no. 17, pp. 1306 $-1308,2015$.

[13] D. E. Anagnostou, A. A. Gheethan, A. K. Amert, and K. W. Whites, "A direct-write printed antenna on paper-based organic substrate for flexible displays and wlan applications," Journal of Display Technology, vol. 6, no. 11 , pp. $558-564,2010$

[14] A. Chen, D. Madan, P. K. Wright, and J. W. Evans, "Dispenserprinted planar thick-film thermoelectric energy generators," Journal of Micromechanics and Microengineering, vol. 21, 2011. 